\title{
A NOTE ON DIFFERENCE SETS
}

D. R. HUGHES

1. Introduction. In [1] R. H. Bruck develops a theory of difference sets in groups that are not necessarily cyclic. In this note we shall present examples of such difference sets (where $\lambda=1$ ) in not-abelian groups of countably infinite order; to do this we generalize a method used by M. Hall in [2]. (For terminology see [1].) The author wishes to thank R. H. Bruck and R. P. Goblirsch for helpful comments.

2. Construction of difference sets. Suppose $G$ is a group, $D$ a subset of $G$, and for every $g \in G, g \neq 1$, there exists exactly one pair $d_{1}, d_{2} \in D$ such that $g=d_{1} d_{2}^{-1}$, and there exists exactly one pair $d_{3}, d_{4} \in D$ such that $g=d_{3}^{-1} d_{4}$. Then $D$ is a 1 -difference set, or merely a difference set, for the group $G$.

Lemмa. Let $G$ be a group and let $S$ be a subset of $G$; then $S$ satisfies (i) if and only if it satisfies (ii).

$$
\begin{array}{llll}
s_{1} s_{2}^{-1}=s_{3} s_{4}^{-1} \neq 1, & s_{i} \in S, & \text { implies } & s_{1}=s_{3}, s_{2}=s_{4} . \\
s_{1}^{-1} s_{2}=s_{3}^{-1} s_{4} \neq 1, & s_{i} \in S, & \text { implies } & s_{1}=s_{3}, s_{2}=s_{4} .
\end{array}
$$

Proof. Suppose $S$ satisfies (i), and $s_{1}^{-1} s_{2}=s_{3}^{-1} s_{4} \neq 1$, where the $s_{i}$ are in $S$. Then $s_{3} s_{1}^{-1}=s_{4} s_{2}^{-1}$. If $s_{1}=s_{3}$, then $s_{2}=s_{4}$, and we have (ii); if $s_{1} \neq s_{3}$, then $s_{3} s_{1}^{-1} \neq 1$, so by (i) we have $s_{3}=s_{4}, s_{1}=s_{2}$, which contradicts $s_{1}^{-1} s_{2} \neq 1$. The other half of the proof is completely similar.

Now let $B$ be a countably infinite group satisfying:

(a) any equation $x^{2}=b$ has at most finitely many solutions $x \in B$ for a given $b \in B$;

(b) $B$ contains no elements of order two;

(c) every element not in the center of $B$ has infinitely many distinct conjugates.

Suppose $D^{\prime}$ is a finite subset of $B$ such that all the quantities $d_{1} d_{2}^{-1}$, for $d_{1}, d_{2} \in D^{\prime}, d_{1} \neq d_{2}$, are distinct (whence all the quantities $d_{1}^{-1} d_{2}$, for $d_{1}, d_{2} \in D^{\prime}, d_{1} \neq d_{2}$, are distinct). Then we shall call $D^{\prime}$ a partial difference set. Given a partial difference set $D^{\prime}$ (possibly empty) and given an element $b \in B$ such that $b \neq d_{1} d_{2}^{-1}$ for any $d_{1}, d_{2} \in D^{\prime}$, we shall extend $D^{\prime}$ to a partial difference set $D^{\prime \prime}$ in which $b=d_{1} d_{2}^{-1}$ holds for some pair $d_{1}, d_{2} \in D^{\prime \prime}$. Then given an element $c \in B$ such that $c \neq d_{1}^{-1} d_{2}$ for any $d_{1}, d_{2} \in D^{\prime \prime}$, we shall extend $D^{\prime \prime}$ to a partial difference set $D^{\prime \prime \prime}$ in which $c=d_{1}^{-1} d_{2}$ holds for some pair $d_{1}, d_{2} \in D^{\prime \prime \prime}$.

Received by the editors December 9, 1954. 
If we show that this can be done, then we can clearly construct a difference set $D$ for the group $B$, since $B$ is countable.

Given $b \in B$ as in the above paragraph, note that $b \neq 1$. Letting $x$ be an arbitrary element of $B$, consider the elements:

$$
x d_{1}^{-1}, d_{1} x^{-1}, b x d_{1}^{-1}, d_{1} x^{-1} b^{-1}, b, b^{-1}, d_{1} d_{2}^{-1} \text {, where } d_{1}, d_{2} \in D^{\prime}, d_{1} \neq d_{2} .
$$

We note that $b \neq b^{-1}$, and $b \neq d_{1} d_{2}^{-1}$; thus the elements of (1) are distinct from one another and from the identity, unless at least one of the following holds:
(1.1) $x d_{1}^{-1} x=d_{2}$;
(1.2) $x d_{1}^{-1} b x=d_{2}$;
(1.3) $x d_{1}^{-1} b x=b^{-1} d_{2}$;

$$
\begin{aligned}
& x=b^{-1} d_{1} d_{2}^{-1} d_{3} ; \\
& x=b^{-1} d_{1} \\
& x=b^{-2} d_{1}
\end{aligned}
$$$$
\text { (1.10) } x^{-1} b x=d_{1}^{-1} d_{2} \text {; }
$$

$$
x=d_{1} d_{2}^{-1} d_{3}
$$$$
x=b d_{1} \text {; }
$$$$
x=d_{1} \text {; }
$$
where $d_{i} \in D^{\prime}$.

Equations (1.4)-(1.9) are satisfied for only finitely many $x$. Equations $(1.1)-(1.3)$ are all of the form $x a x=c$, or $(a x)^{2}=a c$; by hypothesis on $B$, only finitely many $x$ satisfy (1.1)-(1.3).

Now consider (1.10). If $b$ is in the center of $B$, this becomes $b=d_{1}^{-1} d_{2}$, or $d_{1} b=b d_{1}=d_{2}$, or $b=d_{2} d_{1}^{-1}$; so (1.10) is not satisfied at all if $b$ is in the center. If $b$ is not in the center, then $b$ has infinitely many distinct conjugates, so (1.10) is false for infinitely many values of $x$.

Thus we can choose $x$ (in infinitely many ways) so that all the elements of (1) are distinct, and none is the identity. If, for such an $x$, we let $D^{\prime \prime}$ be the set union of $D^{\prime}$ and $x$ and $b x$, then (1) is the set of all differences $d_{1} d_{2}^{-1}$, for $d_{1}, d_{2} \in D^{\prime \prime}, d_{1} \neq d_{2}$. Hence $D^{\prime \prime}$ is a partial difference set, and $b=d_{1} d_{2}^{-1}$ holds for a pair $d_{1}, d_{2} \in D^{\prime \prime}$.

Now if $c \neq d_{1}^{-1} d_{2}$ for any $d_{1}, d_{2} \in D^{\prime \prime}$, we can use a similar process to construct a partial difference set $D^{\prime \prime \prime}$ in which $c=d_{1}^{-1} d_{2}$ holds for some pair $d_{1}, d_{2} \in D^{\prime \prime \prime}$.

Thus we can construct a difference set $D$ for the group $B$.

Condition (b) is necessary in any group $B$ which contains a difference set $D$. For if $b^{2}=1, b \neq 1$, then $b=d_{1} d_{2}^{-1}$ for a unique pair $d_{1}, d_{2} \in D$; thus $b=b^{-1}=d_{2} d_{1}^{-1}$, so $d_{1}=d_{2}$ and $b=1$, a contradiction.

3. Not-abelian free groups. We now show that the not-abelian free group $G$ with $n$ generators ( $n \geqq 2$ ) satisfies the conditions (a), (b), (c) of the preceding section.

Suppose $x=g$, where $g$ is a generator or the inverse of a generator, and $x^{2}=c \neq 1$. If $y=h_{1} h_{2} \cdots h_{m}$ is a reduced form for $y$, and $y^{2}=c$, 
then if $m>1$, there must be a reduction in $h_{1} h_{2} \cdots h_{m} h_{1} h_{2} \cdots h_{m}$; in particular, $h_{m} h_{1}=1$. The reduction must lead to $y^{2}=h_{1} h_{m}=g g$, so $h_{1}=h_{m}=g$, which contradicts $h_{m} h_{1}=1$. So $m=1$, whence clearly $y=x$.

Now suppose $x=g_{1} g_{2}$ is a reduced form for $x$, where each $g_{i}$ is a generator or the inverse of a generator, and $x^{2}=c \neq 1$. Then $c=g_{1} g_{2} g_{1} g_{2}$ and if this is not a reduced form for $c$, then $g_{2} g_{1}=1$ and $x=1$, a contradiction.

If $y=h_{1} h_{2} \cdots h_{m}$ is a reduced form for $y$, and if $y^{2}=c$, then $h_{1} h_{2} \cdots h_{m} h_{1} h_{2} \cdots h_{m}=g_{1} g_{2} g_{1} g_{2}$; this must reduce to $h_{1} h_{2} h_{m-1} h_{m}$ $=g_{1} g_{2} g_{1} g_{2}$, whence $h_{1}=h_{m-1}=g_{1}$ and $h_{2}=h_{m}=g_{2}$. If $m=2$ then it is clear that $y=x$. If $m>2$, then there was a reduction in the first expression for $y^{2}$, and in particular, $h_{m} h_{1}=1$; thus $g_{2} g_{1}=1$ and $x=1$, a contradiction.

Inductively, assume that if the equation $z^{2}=c$, for any $c \in G, c \neq 1$, has a solution $x$ of length $<k$, then the solution is unique. Suppose $x=g_{1} g_{2} \cdots g_{k}$ is a reduced form for $x$, and $x^{2}=c \neq 1$. Suppose $y=h_{1} h_{2} \cdots h_{m}$, where $m \geqq k$, is a reduced form for $y$, and $y^{2}=c$. Then:

$$
h_{1} h_{2} \cdots h_{m} h_{1} h_{2} \cdots h_{m}=g_{1} g_{2} \cdots g_{k} g_{1} g_{2} \cdots g_{k} \text {. }
$$

In all cases, this implies $h_{1}=g_{1}, h_{m}=g_{k}$.

If the right side of (2) is a reduced form for $c$, and if $m=k$, then clearly $y=x$. If $m>k$ then there must be a reduction on the left side of (2), and in particular, $h_{m} h_{1}=1$. But this implies $g_{k} g_{1}=1$, contradicting the assumption that the right side of (2) is a reduced form for $c$. So in this case, $y=x$.

If the right side of (2) is not a reduced form for $c$, then there is a reduction on the right side of (2), so $g_{k} g_{1}=h_{m} h_{1}=1$. Let $x^{\prime}=g_{2} \cdots g_{k-1}$, $y^{\prime}=h_{2} \cdots h_{m-1}$. Equation (2) becomes $x^{\prime 2}=y^{\prime 2}$, where $x^{\prime}$ has length $k-2$. By the induction hypothesis this implies $x^{\prime}=y^{\prime}$, so $x=g_{1} x^{\prime} g_{k}$ $=h_{1} y^{\prime} h_{m}=y$.

Thus (a) holds in $G$.

If $b$ is any element of $G, b \neq 1$, then $b=g_{1}, b=g_{1} g_{2}$, or $b=g_{1} w g_{2}$, where each $g_{i}$ is a generator or the inverse of a generator, and where $b$ is in reduced form.

If $b=g_{1}$, then there is a generator $g$ such that $g \neq g_{1}, g \neq g_{1}^{-1}$. All the elements $g^{-k} b g^{k}$, as $k$ ranges over the integers, are distinct and so $b$ has infinitely many distinct conjugates.

If $b=g_{1} g_{2}$ or $b=g_{1} w g_{2}$, and if $g_{1}=g_{2}$ or $g_{1}=g_{2}^{-1}$, then there is a generator $g$ such that $g \neq g_{1}, g \neq g_{2}$, and hence all the elements $g^{-k} b g^{k}$, as $k$ ranges over the integers, are distinct; so $b$ has infinitely many distinct conjugates. If $g_{1} \neq g_{2}, g_{1} \neq g_{2}^{-1}$, then all the elements $g_{2}^{-k} b g_{2}^{k}$, as 
$k$ ranges over the positive integers, are distinct, so $b$ has infinitely many distinct conjugates.

Thus (c) holds in $G$; it is well known that $G$ satisfies (b).

\section{BIBLIOGRAPHY}

1. R. H. Bruck, Difference sets in a finite group, Trans. Amer. Math. Soc. vol. 78 (1955) pp. 464-481.

2. M. Hall, Cyclic projective planes, Duke Math. J. vol. 14 (1947) pp. 1079-1090.

The University of Wisconsin

\section{MAXIMAL SUBALGEBRAS OF GROUP-ALGEBRAS}

JOHN WERMER

A closed subalgebra of a Banach algebra is called maximal if it is not contained in any larger proper closed subalgebra. Let $G$ be a discrete abelian topological group and $L$ its group-algebra, i.e. $L$ is the Banach algebra of functions $f$ on $G$ with $\sum_{\lambda \in G}|f(\lambda)|<\infty$ and multiplication defined as convolution. What are the maximal subalgebras of $L$ ? The complete answer is not known even when $G$ is the group of integers.

Here we assume that $G$ is ordered. Let $G^{+}$be the semi-group of nonnegative elements of $G$ and $L^{+}$the subset of $L$ consisting of functions which vanish outside of $G^{+}$. Then $L^{+}$is a proper closed subalgebra of $L$.

THEOREM $1 .^{1} L^{+}$is a maximal subalgebra of $L$ if and only if the ordering of $G$ is archimedean.

Proof. Suppose the ordering is non-archimedean. Then we can find $a, b$ in $G^{+}$with $n a<b$ for $n=1,2, \cdots$. Consider the set $G_{1}$ of all elements of $G$ of the form $g^{+}+n(-a)$, where $n=0,1,2, \cdots$ and $g^{+}$is in $G^{+}$. Clearly $G_{1}$ is a semi-group containing $G^{+}$and also $-a$ is in $G_{1}$ and $-b$ is not in $G_{1}$. Let $L_{1}$ be the closed subalgebra of $L$ consisting of all functions vanishing outside $G_{1}$. Then $L_{1}$ lies properly between $L^{+}$and $L$, whence $L^{+}$is not maximal.

Suppose now that the ordering of $G$ is archimedean. Let $\mathfrak{A}^{\prime}$ be a proper closed subalgebra of $L$ with $L^{+}$included in $\mathfrak{A}^{\prime}$. We shall show $\mathfrak{A}^{\prime}=L^{+}$.

Let $E_{\lambda}$ be the function in $L$ with $E_{\lambda}(g)=0, g \neq \lambda, E_{\lambda}(\lambda)=1$. Then

Received by the editors December 27, 1954.

${ }^{1}$ A proof of this theorem has also been found by I. M. Singer. See the note below. 